\title{
Evolución Genética del Virus Influenza A(H1N1) pdm09 en Paraguay 2009-2016
}

\section{Genetic Evolution of the Influenza A (H1N1)pdm09 Virus in Paraguay 2009-2016}

\author{
Cynthia Vázquez ${ }^{1}$ \\ Shirley Villalba ${ }^{1}$ \\ María José Ortega ${ }^{1}$ \\ Andrea Gómez de la Fuente ${ }^{1}$ \\ María Liz Gamarra ${ }^{1}$ \\ Angélica Oviedo ${ }^{1}$ \\ Juan Torales ${ }^{1}$ \\ Marta Von Horoch ${ }^{2}$ \\ 1. Laboratorio Central de Salud Pública \\ 2. Dirección General de Vigilancia De La Salud
}

Una cepa triple reasortante del virus Influenza A emergió en al año 2009 dando origen a una pandemia que alcanzó a Paraguay en junio del mismo año. Con el fin de investigar la evolución genética del virus influenza A (H1N1) pdm09 en Paraguay fueron analizadas las secuencias nucleotídicas del Gen de la Hemaglutinina de 20 cepas de Influenza $\mathrm{A}(\mathrm{H} 1 \mathrm{~N} 1)$ pdm09, aisladas en el Centro Nacional de Influenza de Paraguay entre los años 2009 y 2016, y secuenciadas en el Centro Colaborador de OPS/OMS en Atlanta USA. EI análisis filogenético muestra la circulación de al menos 5 grupos genéticos bien diferenciados de Influenza $\mathrm{A}(\mathrm{H} 1 \mathrm{~N} 1)$ pdm09 en Paraguay desde el 2009. Solamente los virus aislados en el 2016 pertenecen al sub Grupo genético 6B.1 en el cual se encuentra la actual cepa vacunal A/Michigan/45/2015 recomendada para el hemisferio Sur desde el año 2017. Los virus circulantes en años anteriores pertenecen a grupos antigénicamente indistinguibles de la cepa vacunal previa A/California/7/2009. No se encontraron diferencias resaltantes en las secuencias de los virus, relacionadas a severidad clínica ni a distribución geográfica. Los resultados de este estudio reafirman la necesidad de una vigilancia virológica sistemática para orientar el establecimiento de estrategias adecuadas de prevención y control de la influenza

Palabras clave: Cepa vacunal, filogenia, vigilancia virológica.

\section{Abstract}

A triple reassortant strain of Influenza A virus emerged in 2009, leading to a pandemic that reached Paraguay by June the same year. In order to investigate the genetic evolution of influenza A (H1N1)pdm09 virus in Paraguay, we analized the nucleotide sequences of the Hemagglutinin gene of 20 Influenza A (H1N1)pdm09 strains, isolated at the Paraguayan National Influenza Centre between 2009 and 2016, and sequenced at the PAHO/WHO Collaborating Center in Atlanta, USA. Phylogenetic analysis shows the 
circulation of at least 5 well-differentiated genetic groups of Influenza A (H1N1) pdm09 in Paraguay since 2009. Only the viruses isolated in 2016 belong to genetic subgroup 6B.1, the same as the current vaccine strain A/Michigan/45/2015, recommended for the Southern hemisphere since 2017. The viruses circulated previous years belong to groups antigenically indistinguishable from the previous vaccine strain $\mathrm{A} / \mathrm{California/7/2009}$. No significant differences were found in sequences of the viruses, related to clinical severity or geographic distribution. The results of this study reaffirm the need for systematic virological surveillance to guide the establishment of adequate strategies for the prevention and control of influenza

Key words: Vaccine strain, phylogeny, virological surveillance

Fecha de recepción: 09/10/2017

Fecha de aceptación: 15/11/2017

Correspondencia:

Dra. Cynthia Vázquez cynthiavlm@yahoo.com

Laboratorio Central de Salud Pública

\section{Introducción}

Los virus de la influenza (VI) sufren continuamente cambios genéticos, ya sea mediante sustituciones puntuales de aminoácidos (aas) denominados deriva antigénica, o por intercambio de segmentos completos, para evadir la presión del sistema inmune del huésped y adaptarse a nuevos huéspedes ${ }^{1}$. En el año 2009, la circulación de una cepa triple reasortante de Influenza A (A/H1N1pdm2009), desplazó a las previamente circulantes, y ha estado evolucionando continuamente desde entonces hasta la actualidad $^{2-4}$. Los cambios experimentados en los aas del virus pueden alterar sus características antigénicas, su virulencia y su sensibilidad a los antivirales ${ }^{5}$.

La infección por Influenza A(H1N1)pdm09 es principalmente una enfermedad del tracto respiratorio superior leve y autolimitante. El espectro de presentación clínica varía desde casos asintomáticos hasta neumonía viral primaria que resulta en dificultad respiratoria aguda, falla multiorgánica y muerte. ${ }^{6}$ La patogenicidad del virus de la influenza depende de la función de las proteínas virales y de la respuesta inmune del huésped, ${ }^{7}$ de modo que tanto factores virales como del huésped inciden en la patogénesis de la gripe.

El análisis filogenético basado en los cambios de nucleótidos que conducen a sustituciones de aas en las proteínas de superficie Hemaglutinina (HA) y Neuraminidasa (NA) del VI, en conjunto con los análisis antigénicos, son realizados como parte de la vigilancia virológica, a fin de monitorear el impacto en la severidad de los casos y realizar recomendaciones apropiadas sobre las cepas a ser incluidas en las vacunas anuales ${ }^{8}$. Desde su primera detección en Paraguay, en junio de 2009, el VI A(H1N1)pdm09 ha circulado en el país todas las temporadas siguientes, ocasionando tanto casos de enfermedad tipo influenza (ETI) en pacientes ambulatorios como Infecciones respiratorias agudas graves (IRAG) en hospitalizados.

El objetivo de este trabajo es estudiar la diversidad genética del segmento HA de los virus influenza A (H1N1) 2009 circulantes en Paraguay entre 2009 y 2016 


\section{Materiales y métodos}

Se estudiaron muestras representativas de pacientes provenientes de diferentes regiones del Paraguay, captados por la Vigilancia Centinela de ETI e IRAG entre los años 2009 y 2016 . Fueron seleccionados 20 pacientes con diagnóstico confirmado de VI $\mathrm{A}(\mathrm{H} 1 \mathrm{N1}$ )pdm09, 11 de ellos ambulatorios con ETI y 9 hospitalizados con IRAG, a fin de investigar diferencias en la secuencia genómica de los virus aislados a partir de sus muestras. Los datos clínicos y demográficos de los pacientes fueron obtenidos de las fichas epidemiológicas completadas en los centros asistenciales.

Fueron analizadas las secuencias nucleotídicas del Gen HA de las 20 cepas de VI $\mathrm{A}(\mathrm{H} 1 \mathrm{~N} 1)$ pdm09, detectadas por RT-PCR en Tiempo Real y aisladas en el Centro Nacional de Influenza (CNI) de Paraguay entre los años 2009 a 2016; y posteriormente secuenciadas en el Centro Colaborador de OPS/OMS en Atlanta USA (CDC). Todas las secuencias, incluidas las de referencia fueron tomadas de la base de datos "Global Initiative on Sharing All Influenza Data" (GISAID EpiFlu ${ }^{\mathrm{TM}}$ ). Las secuencias fueron alineadas con cepas de referencia y editadas mediante el programa Bioedit y la aplicación Muscle. El análisis filogenético fue realizado por el método neighbor-joining utilizando el programa MEGA $6^{9}$. Las sustituciones de aminoácidos y variaciones en los sitios de glicosilación fueron tabuladas utilizando la aplicación Cubit, comparando con el virus vacunal A/California/07/2009 (H1N1).

\section{Resultados}

Los datos de procedencia y hospitalización de los pacientes estudiados se describen en la tabla 1.

Tabla 1. Datos de procedencia y hospitalización de los pacientes estudiados. Paraguay $2009-2016$ 


\begin{tabular}{|c|c|c|c|}
\hline AÑO & $\begin{array}{c}\text { Región } \\
\text { Sanitaria }\end{array}$ & $\begin{array}{c}\mathbf{N}^{*} \text { de Pacientes } \\
\text { Ambulatorios }\end{array}$ & $\begin{array}{c}\mathbf{N}^{\circ} \text { de Pacientes } \\
\text { Hospitalizados }\end{array}$ \\
\hline \multirow{2}{*}{2009} & Central & 2 & \\
\hline & Asunción & 1 & \\
\hline \multirow{2}{*}{2010} & Caazapa & & 1 \\
\hline & Guaira & 1 & \\
\hline \multirow{2}{*}{2011} & Asunción & 1 & \\
\hline & Concepción & & 1 \\
\hline \multirow{2}{*}{2012} & Central & & 1 \\
\hline & Asunción & & 1 \\
\hline \multirow{2}{*}{2013} & Central & 1 & \\
\hline & Alto Paraná & & 1 \\
\hline \multirow{2}{*}{2014} & Asunción & & 1 \\
\hline & Amambay & 1 & \\
\hline \multirow{3}{*}{2015} & Asunción & 2 & \\
\hline & Central & 1 & \\
\hline & Caaguazu & & 1 \\
\hline \multirow{3}{*}{2016} & Asunción & & 1 \\
\hline & Central & 1 & \\
\hline & Caaguazu & & 1 \\
\hline \multicolumn{2}{|c|}{ TOTAL } & 11 & 9 \\
\hline
\end{tabular}

El análisis filogenético muestra la circulación de cepas pertenecientes al menos a 5 grupos genéticos (5, 6, 6B, 6C, 6B.1) de VI A(H1N1)pdm09 en Paraguay desde el 2009 (Figura1). 


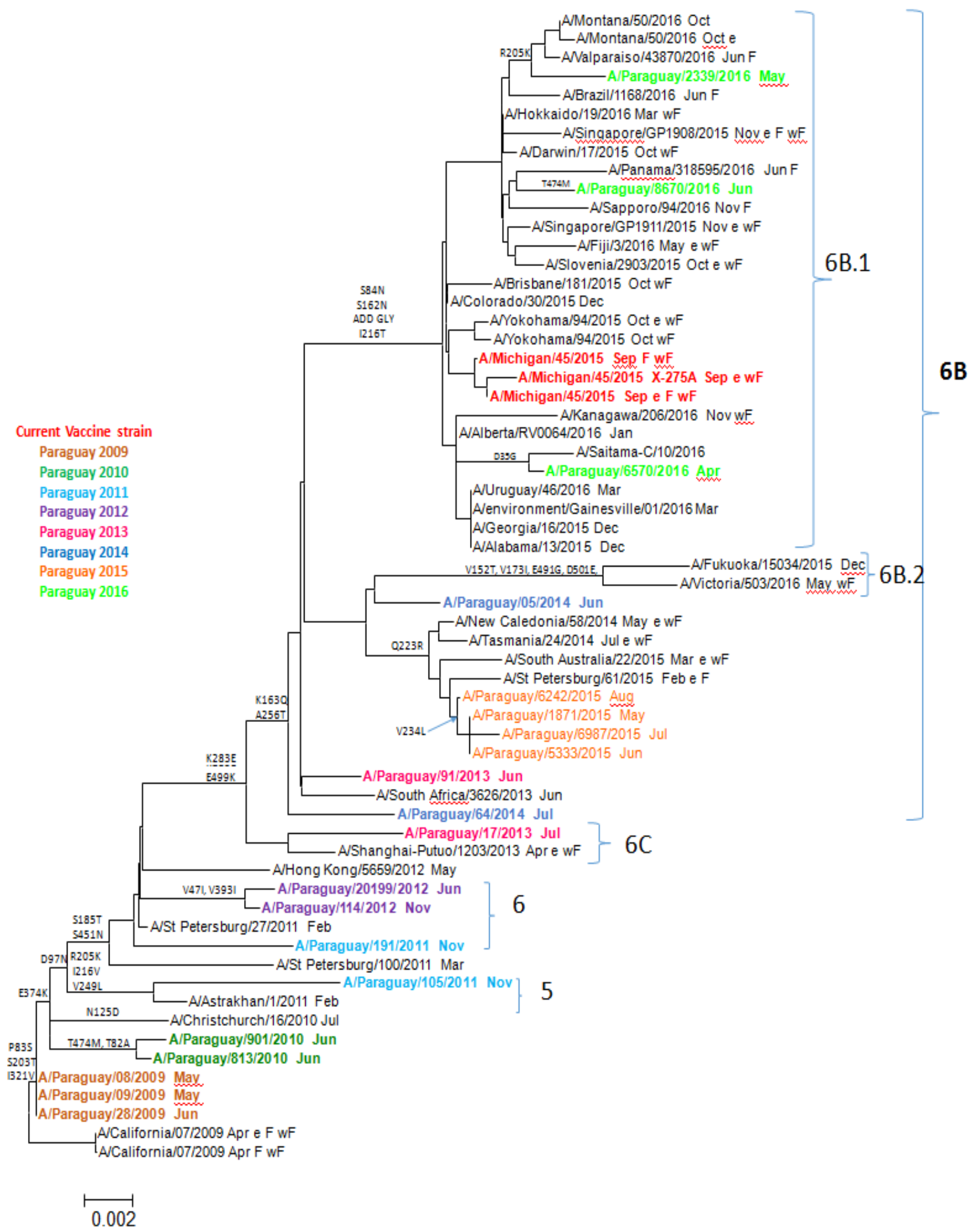

Figura 1. Relaciones evolutivas entre la gripe $A(\mathrm{H} 1 \mathrm{~N} 1)$ pdm09 Gen de hemaglutinina (HA). Paraguay 2009-2016

Los virus del 2009 fueron muy cercanos al virus de referencia A/California/07/2009 (H1N1), con solo 3 sustituciones de aas (P83S, S203T, I132V). Se nota una tendencia a incrementar progresivamente la frecuencia del número acumulado de sustituciones de aas 
las siguientes temporadas de influenza, con 6 y 8 sustituciones en el 2010, entre las que destacan como nuevas: E374K, T474M, T82A. (Tabla 2).

Tabla 2. Sustituciones de aminoácidos en el segmento HA de los virus Influenza A(H1N1)pdm09 circulantes en Paraguay 2009-2016

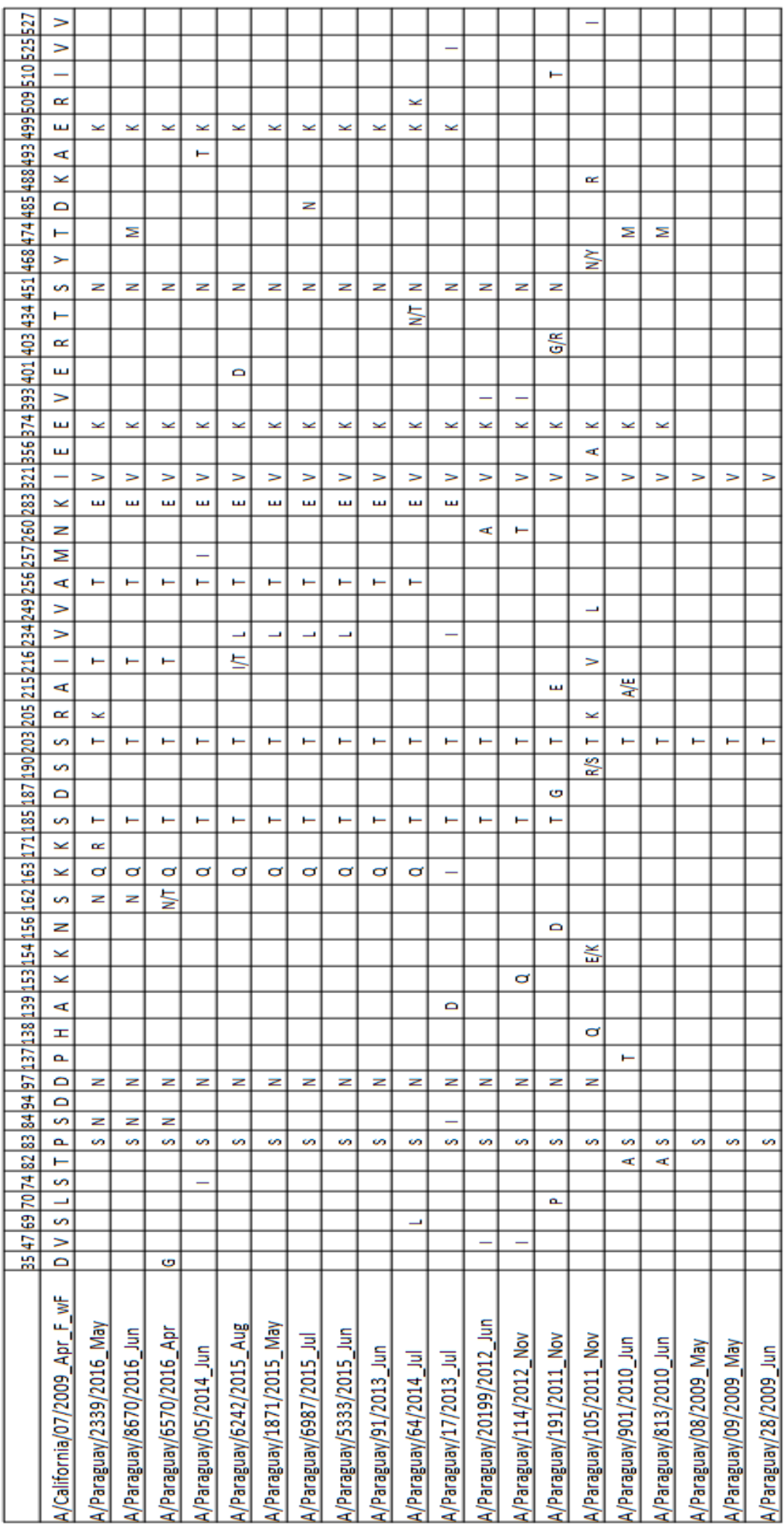

las cepas pertenece al Grupo 5, caracterizado 
sustituciones R205K, I216V, V249L. Otra cepa del 2011 y las del 2012 pertenecen al Grupo 6 caracterizado por las sustituciones S185T y S451N, además todos presentan la mutación D97N. Las cepas del 2012 presentan las sustituciones adicionales V47I y V393I. Uno de los virus aislados en 2013 pertenece al grupo 6C caracterizado por las sustituciones de aas K283E y E499K; mientras que otro virus del 2013 al igual que los del 2014 y 2015 quedaron en el Grupo 6B caracterizado por las sustituciones K163Q y A256T. Solamente los virus aislados en 2016 pertenecen al sub Grupo genético 6B.1 con la característica sustitución S162N que agrega un motivo de glicosilación (Tabla 3), además de las sustituciones en S84N y I216T. En este mismo grupo se encuentra la actual cepa vacunal A/Michigan/45/2015.

Tabla 3. Cambios en los Sitios de glicosilación del segmento HA de los virus Influenza A(H1N1)pdm09 circulantes en Paraguay 2009-2016

\begin{tabular}{|c|c|c|c|c|c|c|c|c|c|c|c|}
\hline CEPA VIRAL & \begin{tabular}{c|} 
SITIOS DE \\
GLICOSILACION
\end{tabular} & 10 & 11 & 23 & 87 & 119 & 162 & 276 & 287 & 481 & 540 \\
\hline A/California/07/2009_Apr_F_wF_8gs & \begin{tabular}{|l|}
8 \\
\end{tabular} & $\mathrm{x}$ & $\mathrm{x}$ & $\mathrm{x}$ & $\mathrm{x}$ & & & $\mathrm{x}$ & $\mathrm{x}$ & $\mathrm{x}$ & $\mathrm{x}$ \\
\hline A/Paraguay/2339/2016_May_9gs & 9 & $\mathrm{x}$ & $\mathrm{x}$ & $\mathrm{x}$ & $\mathrm{x}$ & & $\mathrm{x}$ & $\mathrm{x}$ & $\mathrm{x}$ & $x$ & $\mathrm{x}$ \\
\hline A/Paraguay/8670/2016_Jun_9gs & 9 & $\mathrm{x}$ & $\mathrm{x}$ & $\mathrm{x}$ & $\mathrm{x}$ & & $\mathrm{x}$ & $\mathrm{x}$ & $\mathrm{x}$ & $\mathrm{x}$ & $\mathrm{x}$ \\
\hline A/Paraguay/6570/2016_Apr_9gs & 9 & $\mathrm{x}$ & $\mathrm{x}$ & $\mathrm{x}$ & $\mathrm{x}$ & & $\mathrm{x}$ & $\mathrm{x}$ & $\mathrm{x}$ & $\mathrm{x}$ & $\mathrm{x}$ \\
\hline A/Paraguay/05/2014_Jun_8gs & 8 & $\mathrm{x}$ & $\mathrm{x}$ & $\mathrm{x}$ & $\mathrm{x}$ & & & $\mathrm{x}$ & $\mathrm{x}$ & $\mathrm{x}$ & $\mathrm{x}$ \\
\hline A/Paraguay/6242/2015_Aug_8gs & 8 & $\mathrm{x}$ & $\mathrm{x}$ & $\mathrm{x}$ & $\mathrm{x}$ & & & $\mathrm{x}$ & $\mathrm{x}$ & $\mathrm{x}$ & $\mathrm{x}$ \\
\hline A/Paraguay/1871/2015_May_8gs & 8 & $\mathrm{x}$ & $\mathrm{x}$ & $x$ & $\mathrm{x}$ & & & $x$ & $x$ & $\mathrm{x}$ & $\mathrm{x}$ \\
\hline A/Paraguay/6987/2015_Jul_8gs & 8 & $\mathrm{x}$ & $\mathrm{x}$ & $x$ & $\mathrm{x}$ & & & $\mathrm{x}$ & $x$ & $\mathrm{x}$ & $\mathrm{x}$ \\
\hline A/Paraguay/5333/2015_Jun_8gs & 8 & $\mathrm{x}$ & $\mathrm{x}$ & $x$ & $\mathrm{x}$ & & & $\mathrm{x}$ & $\mathrm{x}$ & $\mathrm{x}$ & $\mathrm{x}$ \\
\hline A/Paraguay/91/2013_Jun_8gs & 8 & $\mathrm{x}$ & $\mathrm{x}$ & $\mathrm{x}$ & $\mathrm{x}$ & & & $\mathrm{x}$ & $\mathrm{x}$ & $\mathrm{x}$ & $\mathrm{x}$ \\
\hline A/Paraguay/64/2014_Jul_8gs & 8 & $\mathrm{x}$ & $\mathrm{x}$ & $\mathrm{x}$ & $\mathrm{x}$ & & & $\mathrm{x}$ & $\mathrm{x}$ & $\mathrm{x}$ & $\mathrm{x}$ \\
\hline A/Paraguay/17/2013_Jul_8gs & 8 & $\mathrm{x}$ & $\mathrm{x}$ & $x$ & $\mathrm{x}$ & & & $\mathrm{x}$ & $x$ & $\mathrm{x}$ & $\mathrm{x}$ \\
\hline A/Paraguay/20199/2012_Jun_8gs & 8 & $\mathrm{x}$ & $\mathrm{x}$ & $x$ & $\mathrm{x}$ & & & $\mathrm{x}$ & $\mathrm{x}$ & $\mathrm{x}$ & $\mathrm{x}$ \\
\hline A/Paraguay/114/2012_Nov_8gs & 8 & $\mathrm{x}$ & $\mathrm{x}$ & $\mathrm{x}$ & $\mathrm{x}$ & & & $\mathrm{x}$ & $\mathrm{x}$ & $\mathrm{x}$ & $\mathrm{x}$ \\
\hline A/Paraguay/191/2011_Nov_8gs & 8 & $\mathrm{x}$ & $\mathrm{x}$ & $\mathrm{x}$ & $\mathrm{x}$ & & & $\mathrm{x}$ & $\mathrm{x}$ & $\mathrm{x}$ & $\mathrm{x}$ \\
\hline A/Paraguay/105/2011_Nov_8gs & 8 & $\mathrm{x}$ & $\mathrm{x}$ & $x$ & $\mathrm{x}$ & & & $\mathrm{x}$ & $\mathrm{x}$ & $\mathrm{x}$ & $\mathrm{x}$ \\
\hline A/Paraguay/901/2010_Jun_8gs & 8 & $\mathrm{x}$ & $\mathrm{x}$ & $x$ & $\mathrm{x}$ & & & $\mathrm{x}$ & $\mathrm{x}$ & $\mathrm{x}$ & $\mathrm{x}$ \\
\hline A/Paraguay/813/2010_Jun_8gs & 8 & $\mathrm{x}$ & $\mathrm{x}$ & $\mathrm{x}$ & $\mathrm{x}$ & & & $\mathrm{x}$ & $\mathrm{x}$ & $\mathrm{x}$ & $\mathrm{x}$ \\
\hline A/Paraguay/08/2009_May_8gs & 8 & $\mathrm{x}$ & $\mathrm{x}$ & $\mathrm{x}$ & $\mathrm{x}$ & & & $\mathrm{x}$ & $\mathrm{x}$ & $\mathrm{x}$ & $\mathrm{x}$ \\
\hline A/Paraguay/09/2009_May_8gs & 8 & $\mathrm{x}$ & $\mathrm{x}$ & $x$ & $\mathrm{x}$ & & & $\mathrm{x}$ & $x$ & $\mathrm{x}$ & $\mathrm{x}$ \\
\hline A/Paraguay/28/2009_Jun_8gs & 8 & $\mathrm{x}$ & $\mathrm{x}$ & $\mathrm{x}$ & $\mathrm{x}$ & & & $\mathrm{x}$ & $\mathrm{x}$ & $\mathrm{x}$ & $\mathrm{x}$ \\
\hline
\end{tabular}

No se encontraron diferencias resaltantes entre las secuencias de lo virus pertenecientes a pacientes ambulatorios y hospitalizados dentro de cada año. Tampoco se detectaron diferencias importantes según región geográfica dentro del mismo periodo de tiempo.

\section{Discusión}


En el presente estudio hemos identificado al menos 5 grupos genéticos diferentes de VI A(H1N1)pdm09 que circularon en Paraguay desde su primera introducción en junio del 2009 hasta el año 2016. Esta agrupación está basada en variaciones de aas de la HA, que es la glicoproteína de la superficie viral responsable de la unión al receptor de la célula huésped y la posterior fusión de membrana y entrada viral. ${ }^{10}$ La HA juega un papel importante en la respuesta inmune del huésped, estimulando la producción de anticuerpos neutralizantes responsables de la inmunidad protectora ${ }^{11-13}$.

Entre los virus de Paraguay estudiados algunos presentan mutaciones relacionadas a sitios antigénicos como K163Q, S185T, S203T y R205K. Los cambios de aminoácidos que ocurren en los sitios antigénicos o en la superficie de la molécula de HA pueden tener un efecto importante en el reconocimiento de anticuerpos, permitiendo al virus escapar de la respuesta inmune ${ }^{14}$. Entre otras mutaciones relevantes que presentan los virus estudiados, se encuentran las sustituciones de aminoácidos S185T dentro del sitio de unión al receptor, y $1216 \mathrm{~V}$ cerca de del mismo sitio, que pueden afectar la interacción de la HA con su receptor celular ${ }^{15}$.

Ninguno de los virus de Paraguay presento la mutación D222G que ha sido considerado un marcador de virulencia asociado con mayor severidad clínica ${ }^{16}$. Esta mutación ha sido asociada a un aumento de la avidez en la unión viral a receptores celulares que poseen ácido siálico unido a galactosa por un enlace $\alpha 2,3^{17,18}$. Este tipo de receptores se encuentran en la región alveolar de los pulmones humanos lo que podría explicar la neumonía grave, al igual que en los casos humanos de infección H5N1, lo que estaría indicando la existencia de replicación viral en las vías respiratorias inferiores ${ }^{19}$. En concordancia con este hallazgo, los pacientes estudiados no manifestaron diferencias en la severidad clínica atribuibles a mutaciones virales, ya que los virus tanto de los casos ambulatorios como de los hospitalizados presentaron secuencias similares de aas dentro de un mismo periodo de tiempo. Por lo tanto habría que investigar otras causas para la severidad de la infección en algunos de estos pacientes, como comorbilidades o coinfecciones, lo cual escapa a los objetivos de este estudio.

Según estudios realizados en los centros colaboradores de OMS, los VI pertenecientes a todos los grupos genéticos detectados son antigénicamente indistinguibles del virus vacunal A/California/7/2009, a pesar de los cambios en aas y sitios de glicosilación ${ }^{20}$. Sin embargo según las recomendaciones para el hemisferio sur en el 2017, virus representativos del grupo emergente 6B.1, al cual pertenecen los virus de Paraguay aislados en el 2016, fueron poco inhibidos por algunos grupos de sueros humanos adultos posteriores a la vacunación. Además la media de los títulos de inhibición de sueros pediátricos pos vacunación contra algunos virus representativos del grupo 6B.1 se redujeron significativamente en comparación con los títulos contra el virus de la vacuna A/California/7/2009 ${ }^{21}$. Por ese motivo por primera vez desde la pandemia del año 2009, se sustituyó la cepa vacunal A/California/7/2009 por A/Michigan/45/2015. La nueva cepa vacunal pertenece al grupo 6B.1 caracterizado por la sustitución S162N que modifica un sitio de glicosilación, lo que podría conducir a cambios en el reconocimiento antigénico.

El análisis de la evolución genética del VI A(H1N1)pdm09 en Paraguay, indica que recién desde el año 2016 circularon en el país cepas pertenecientes al grupo antigénico emergente al cual pertenece la nueva nueva cepa vacunal recomendada a partir del año 2017. Mientras que los virus circulantes en años anteriores pertenecen a grupos genéticos que resultaron antigénicamente similares a la cepa vacunal previa A/California/7/2009.

\section{Conclusión}


Estos resultados demuestran la importancia de la Vigilancia virológica sistemática y la caracterización genética de los virus de Influenza, en la provisión de datos oportunos para la formulación adecuada de vacunas recomendadas a nuestra región; así como en la detección de cambios en el genoma viral, que podrían estar relacionados a un aumento de la severidad clínica, de manera a alertar al sistema de salud para responder apropiadamente ante los brotes de influenza.

\section{Reconocimientos}

Agradecemos a CDC por el apoyo financiero para la adquisición de reactivos e insumos en el marco del Proyecto "Sostenibilidad de la Vigilancia de Influenza"; a OPS/OMS por el apoyo técnico para la Vigilancia virológica y epidemiológica de Influenza; al Fondo para la Convergencia Estructural de Mercosur (COF 03/11), por el apoyo en la contratación de las investigadoras María Liz Gamarra y Andrea Gómez de la Fuente.

Reconocemos a los autores, laboratorios de origen y laboratorios que remitieron las secuencias disponibles en la base de datos GISAID's EpiFlu ${ }^{\mathrm{TM}}$ en la cual esta investigación está basada. La lista se detalla a continuación.

\begin{tabular}{|c|c|c|c|c|c|c|}
\hline $\begin{array}{l}\text { Segmento } \\
\text { ID }\end{array}$ & Segmento & Pais & Nombre del aislado & Laboratorio de Origen & $\begin{array}{l}\text { Laboratorio } \\
\text { Remitente }\end{array}$ & Autores \\
\hline EPI721763 & HA & Japan & A/SAITAMA-C/ $/ 10 / 2016$ & $\begin{array}{l}\text { Saitama City Institute of Health } \\
\text { Science and Research }\end{array}$ & NIID & $\begin{array}{c}\text { Takashita,Emi; } \\
\text { Fujisaki,Seichiro; } \\
\text { Shirakura,M asayuki; } \\
\text { Wata nabe,Sh inji; } \\
\text { Odagiri,Takato } \\
\end{array}$ \\
\hline EPI828660 & HA & Uruguay & A/U ruguay/46/2016 & $\begin{array}{l}\text { Departamento de Laboratorio } \\
\text { de Salud Pública (DLSP) }\end{array}$ & $\mathrm{CDC}$ & \\
\hline EPI176504 & $\mathrm{HA}$ & United States & A/C alifo mia/07/2009 & \multirow{2}{*}{$\begin{array}{l}\text { Naval Health Research Center } \\
\text { Colorado Department of Health } \\
\text { Lab }\end{array}$} & $\mathrm{CDC}$ & \\
\hline EPI700671 & $\mathrm{HA}$ & United States & A/Colorado/30/2015 & & $\mathrm{CDC}$ & \\
\hline EPI716307 & HA & United States & A/Georgia/16/2015 & $\begin{array}{c}\text { Georgia Public Health } \\
\text { Laboratory }\end{array}$ & $\mathrm{CDC}$ & \\
\hline E PI685436 & HA & United States & A/Alabama/13/2015 & $\begin{array}{c}\text { ADPH Bureau of Clinical } \\
\text { Laboratories }\end{array}$ & CDC & \\
\hline EPI759256 & HA & Canada & A/Alberta/RV0064/2016 & $\begin{array}{c}\text { National Microbiology } \\
\text { Laboratory, Health Canada }\end{array}$ & CDC & \\
\hline EPI842069 & HA & Brazil & A/Brazil1168/2016 & $\begin{array}{l}\text { Instituto O swaldo Cruz } \\
\text { FIOCRUZ - Laboratory of } \\
\text { Respiratory Viruses and } \\
\text { Measles (LVRS) }\end{array}$ & CDC & \\
\hline E PI662594 & HA & United States & A/M ich igan/45/2015 & $\begin{array}{l}\text { M ichigan Department of } \\
\text { Community } \mathrm{He} \text { alth }\end{array}$ & CDC & \\
\hline E PI668427 & HA & United States & A/M ich igan/45/2015 & $\begin{array}{l}\text { M ichigan Department of } \\
\text { Community Health }\end{array}$ & $\mathrm{CDC}$ & \\
\hline EPI849370 & HA & United States & $\begin{array}{l}\text { A/M ichigan/45/2015 X- } \\
275 \mathrm{~A}(16 / 274)\end{array}$ & & NIBSC & Nicolson, Carolyn \\
\hline E PI862979 & HA & United States & A/M ontana/50/2016 & $\begin{array}{c}\text { Montana Laborato ry Services } \\
\text { Bureau }\end{array}$ & CDC & \\
\hline EPI871202 & HA & United States & A/M ontana/50/2016 & $\begin{array}{c}\text { Montana Laboratory Services } \\
\text { Bureau }\end{array}$ & CDC & \\
\hline E PI815676 & HA & Panama & A/Panama/318595/2016 & $\begin{array}{l}\text { Instituto Conmem orativo } \\
\text { Gorgas de Estudios de la }\end{array}$ & $\mathrm{CDC}$ & \\
\hline EPI849443 & HA & Singapore & $\begin{array}{l}\text { A/Sing apore/GP } 1908 / 20 \\
15\end{array}$ & $\begin{array}{l}\text { WHO Collaborating Centre for } \\
\text { Reference and Research on } \\
\text { Influenza }\end{array}$ & CDC & \\
\hline EPI768541 & HA & Slovenia & A/Slovenia/2903/2015 & $\begin{array}{c}\text { National Institute for M edical } \\
\text { Research }\end{array}$ & CDC & \\
\hline EPI625972 & HA & $\begin{array}{c}\text { Russian } \\
\text { Federation }\end{array}$ & A/St P etersburg/61/2015 & $\begin{array}{l}\text { Russian Academy of Medical } \\
\text { Sciences }\end{array}$ & CDC & \\
\hline EPI830111 & HA & Chile & $\begin{array}{c}\text { ANalpa raiso/43870/201 } \\
6 \\
\end{array}$ & $\begin{array}{l}\text { Instituto de Salud P ublica de } \\
\text { Chile }\end{array}$ & $\mathrm{CDC}$ & \\
\hline
\end{tabular}




\begin{tabular}{|c|c|c|c|c|c|c|}
\hline EPI676509 & HA & Japan & AMOКОН АM A/9 4/2015 & $\begin{array}{c}\text { Yokohama City Institute of } \\
\text { Public Health. }\end{array}$ & NIID & $\begin{array}{c}\text { Takashita,Emi; } \\
\text { Fujisaki,Seiichiro; } \\
\text { Shirakura,M asayuki; } \\
\text { Wata nabe,Sh inji; } \\
\text { Odagiri,Takato } \\
\end{array}$ \\
\hline EPI673558 & HA & Japan & AMOKOH AMAV9 4/2015 & $\begin{array}{l}\text { Yokohama City Institute of } \\
\text { Public Health. }\end{array}$ & NIID & $\begin{array}{c}\text { Takashita,Emi; } \\
\text { Fujisaki,Seiichiro; } \\
\text { Shirakura,M asayuki; } \\
\text { Wata nabe,Sh inji; } \\
\text { Odagiri,Takato }\end{array}$ \\
\hline EPI858855 & HA & Slovenia & A/Slovenia/2903/2015 & $\begin{array}{l}\text { Laboratory for Virolo gy, } \\
\text { National Institute of Public } \\
\text { Health }\end{array}$ & $\begin{array}{l}\text { Crick Worldwide } \\
\text { Influenza Centre }\end{array}$ & \\
\hline EPI956861 & HA & Australia & $\begin{array}{c}\text { A/South } \\
\text { Australia/22/2015 }\end{array}$ & $\begin{array}{l}\text { Institute of Medical and } \\
\text { Veterinary Science (IMVS) }\end{array}$ & $\begin{array}{l}\text { WHO Collaborating } \\
\text { Centre for Reference } \\
\text { and Research on } \\
\text { Influenza }\end{array}$ & $\begin{array}{c}\text { Deng, Y-M.; } \\
\text { lanne llo, } P . ; \\
\text { Spira son, } N . ; \text { Lau,H.; } \\
\text { Kaye,M.; } \\
\text { Komadina, } N \text {. } \\
\end{array}$ \\
\hline EPI706473 & HA & Japan & $\begin{array}{c}\text { A/FU KU OKA } 15034 / 201 \\
5\end{array}$ & $\begin{array}{l}\text { Fukuoka Institute of Public } \\
\text { Health and Environm ental } \\
\text { Sciences }\end{array}$ & NIID & $\begin{array}{c}\text { Takashita,Emi; } \\
\text { Fujisaki,Seiichiro; } \\
\text { Shirakura,M asayuki; } \\
\text { Wata nabe,Sh inji; } \\
\text { Odagiri,Takato } \\
\text { Takashita,Emi; }\end{array}$ \\
\hline EPI769352 & HA & Japan & A/HOKKAIDO/19/2016 & $\begin{array}{l}\text { Hokkaid o Institute of Public } \\
\text { Health }\end{array}$ & NIID & $\begin{array}{c}\text { Fujisaki,Seiichiro; } \\
\text { Shirakura,M asayuki; } \\
\text { Wata nabe,Sh inji; } \\
\text { Odagiri,Takato } \\
\end{array}$ \\
\hline E PI917557 & HA & Japan & A/Kanaga va/206/2016 & $\begin{array}{l}\text { Nation al Institute of In fectious } \\
\text { Diseases (NIID) }\end{array}$ & CDC & \\
\hline E PI917659 & HA & Japan & A/Sapporo/94/2016 & $\begin{array}{c}\text { Nation al Institute of Infectious } \\
\text { Diseases (NIID) }\end{array}$ & CDC & \\
\hline EPI704084 & HA & Australia & A/Brisbane/181/2015 & $\begin{array}{c}\text { Queensland Health Scientific } \\
\text { Services }\end{array}$ & $\begin{array}{l}\text { WHO Collaborating } \\
\text { Centre for Reference } \\
\text { and Research on } \\
\text { Influenza }\end{array}$ & $\begin{array}{l}\text { Deng,Y-M.; } \\
\text { lannello,P.; } \\
\text { Spirason, } \mathrm{N}, ; \text { Lau,H.; } \\
\text { Komadina, } \mathrm{N} \\
\end{array}$ \\
\hline E PI675532 & HA & Australia & A/Darwin/17/2015 & Royal Darwin Hospital & $\begin{array}{l}\text { WHO Collaborating } \\
\text { Centre for Reference } \\
\text { and Research on } \\
\text { Influenza }\end{array}$ & $\begin{array}{l}\text { Deng, Y-M.; } \\
\text { lannello,P.; } \\
\text { Spirason, } \mathrm{N} . ; \text { Lau, } \mathrm{H} ; \\
\text { Komadina,N. } \\
\end{array}$ \\
\hline EPI793049 & HA & Fiji & $\mathrm{A} / \mathrm{Fiji} / 3 / 2016$ & $\begin{array}{l}\text { National Centre for Scientific } \\
\text { Services for Virology and } \\
\text { Vector Borne Diseases }\end{array}$ & $\begin{array}{l}\text { WHO Collaborating } \\
\text { Centre for Reference } \\
\text { and Research on } \\
\text { Influenza }\end{array}$ & $\begin{array}{c}\text { Deng, Y-M.; } \\
\text { lanne llo,P.; } \\
\text { Sprirason, } N \text {.; Lau,H.; } \\
\text { Komadina,N. } \\
\end{array}$ \\
\hline EPI746509 & HA & Singapore & $\begin{array}{c}\text { A/Sing apore/GP 1911/20 } \\
15\end{array}$ & Ministry of Health, Sing apore & $\begin{array}{l}\text { WHO Collaborating } \\
\text { Centre for Reference } \\
\text { and Research on } \\
\text { Influenza }\end{array}$ & $\begin{array}{c}\text { Deng, Y-M.; } \\
\text { lann ello,P.; Spirason, } \\
\text { N.; Lau,H.; } \\
\text { Komadina,N. }\end{array}$ \\
\hline E PI956794 & HA & Australia & ANictoria/503/2016 & Monash Medical Centre & $\begin{array}{l}\text { WHO Collaborating } \\
\text { Centre for Reference } \\
\text { and Research on } \\
\text { Influenza }\end{array}$ & $\begin{array}{c}\text { Deng,Y-M.; } \\
\text { lannello,P.; } \\
\text { Spirason, } \mathrm{N}_{\text {. }} ; \text { Lau,H.; } \\
\text { Kaye,M.; } \\
\text { Komadina,N. } \\
\end{array}$ \\
\hline EPI748148 & HA & $\begin{array}{c}\text { New } \\
\text { Caledonia }\end{array}$ & $\begin{array}{c}\text { A/New } \\
\text { Caledonia/58/2014 }\end{array}$ & Institut Pasteur New Cale donia & $\begin{array}{l}\text { WHO Collaborating } \\
\text { Centre for Reference } \\
\text { and Research on } \\
\text { Influenza }\end{array}$ & $\begin{array}{l}\text { Deng,Y-M.; } \\
\text { lannello,P.; } \\
\text { Spirason,N.; Lau,H.; } \\
\text { Komadina,N } \\
\end{array}$ \\
\hline EPI748200 & HA & Australia & A/Tasmania/24/2014 & Royal Hobart Hospital & $\begin{array}{l}\text { WHO Collaborating } \\
\text { Centre for Reference } \\
\text { and Research on } \\
\text { Influenza }\end{array}$ & $\begin{array}{c}\text { Deng,Y-M.; } \\
\text { lannello,P.; } \\
\text { Spira son,N.; Lau,H.; } \\
\text { Komadina,N } \\
\end{array}$ \\
\hline E PI577 031 & HA & South Africa & $\begin{array}{c}\text { A/South } \\
\text { Africa/3626/2013 } \\
\end{array}$ & $\begin{array}{l}\text { National Institute for Medical } \\
\text { Research }\end{array}$ & $\mathrm{CDC}$ & \\
\hline E PI477030 & HA & China & $\begin{array}{c}\text { A/Shan ghai- } \\
\text { Putu 0/1203/2013 } \\
\end{array}$ & $\begin{array}{l}\text { WHO Chinese National } \\
\text { Influenza Center }\end{array}$ & $\begin{array}{l}\text { National Institute for } \\
\text { Medical Research }\end{array}$ & \\
\hline EPI382424 & HA & $\begin{array}{l}\text { Hong Kong } \\
\text { (SAR) }\end{array}$ & A/Hong Kong/5659/2012 & $\begin{array}{l}\text { Hong Kong Department of } \\
\text { Health }\end{array}$ & $\begin{array}{c}\text { Hong Kong } \\
\text { Department of Health }\end{array}$ & Mak,G.C.;Lo,J.Y.C. \\
\hline EPI319527 & HA & $\begin{array}{c}\text { Russian } \\
\text { Federation } \\
\end{array}$ & $\begin{array}{c}\text { A/St. } \\
\text { Petersburg/27/2011 } \\
\end{array}$ & $\begin{array}{l}\text { WHO National Influenza } \\
\text { Centre Russian Federation }\end{array}$ & $\begin{array}{l}\text { National Institute for } \\
\text { Medical Research }\end{array}$ & \\
\hline EPI316435 & HA & $\begin{array}{c}\text { Russian } \\
\text { Federation }\end{array}$ & $\begin{array}{c}\text { A/St. } \\
\text { Petersburg/100/2011 } \\
\end{array}$ & $\begin{array}{l}\text { Russian Academy of M edical } \\
\text { Sciences }\end{array}$ & $\mathrm{CDC}$ & \\
\hline EPI319590 & HA & $\begin{array}{l}\text { Russian } \\
\text { Federation }\end{array}$ & A/Astrakhan/1/2011 & $\begin{array}{l}\text { WHO National Influenza } \\
\text { Centre Russian Federation }\end{array}$ & $\begin{array}{l}\text { National Institute for } \\
\text { Medical Research }\end{array}$ & \\
\hline E PI280344 & HA & New Zealand & A/Christchurch/16/2010 & $\begin{array}{l}\text { WHO Collaborating Centre for } \\
\text { Reference and Research on } \\
\text { Influenza }\end{array}$ & CDC & \\
\hline $\mathrm{EPI} 221023$ & HA & Paraguay & A/Paraguay/08/2009 & $\begin{array}{l}\text { Laboratorio Central de Salud } \\
\text { Pública }\end{array}$ & $\mathrm{CDC}$ & \\
\hline EPI183250 & HA & Paraguay & A/Paraguay/09/2009 & $\begin{array}{l}\text { Laboratorio Central de Salud } \\
\text { Pública }\end{array}$ & $\mathrm{CDC}$ & \\
\hline EPI184390 & HA & Paraguay & A/Paraguay/28/2009 & $\begin{array}{c}\text { Laboratorio Central de Salud } \\
\text { Pública }\end{array}$ & $\mathrm{CDC}$ & \\
\hline
\end{tabular}




\begin{tabular}{|c|c|c|c|c|c|}
\hline EPI301698 & HA & Paraguay & A/Paragu ay/813/2010 & $\begin{array}{l}\text { Laboratorio Central de Salud } \\
\text { Pública }\end{array}$ & CDC \\
\hline EPI301701 & $\mathrm{HA}$ & Paraguay & A/Paraguay/201/2010 & $\begin{array}{l}\text { Laboratoriq, Central de Salud } \\
\text { publica }\end{array}$ & CDC \\
\hline EPI349349 & HA & Paraguay & A/Paraguay/105/2011 & $\begin{array}{l}\text { Laboratorio Central de Salud } \\
\text { Pública }\end{array}$ & CDC \\
\hline EPI349352 & $\mathrm{HA}$ & Paraguay & A/Paraguay/191/2011 & $\begin{array}{l}\text { Laboratorio Central de Salud } \\
\text { Pública }\end{array}$ & CDC \\
\hline EPI387972 & HA & Paraguay & A/Paraguay/114/2012 & $\begin{array}{c}\text { Laboratorio Central de Salud } \\
\text { Pública }\end{array}$ & CDC \\
\hline EPI394758 & $\mathrm{HA}$ & P araguay & A/Paraguay/20199/2012 & $\begin{array}{c}\text { Laboratorio Central de Salud } \\
\text { Pública }\end{array}$ & CDC \\
\hline EPI842146 & HA & Paraguay & A/Paraguay/6570/2016 & $\begin{array}{c}\text { Laboratorio Central de Salud } \\
\text { Pública }\end{array}$ & CDC \\
\hline EPI828436 & HA & Paraguay & A/Paraguay/2339/2016 & $\begin{array}{c}\text { Laboratorio Central de Salud } \\
\text { Pública }\end{array}$ & CDC \\
\hline EPI825445 & HA & Paraguay & A/Paraguay/8670/2016 & $\begin{array}{l}\text { Laboratorio Central de Salud } \\
\text { Pública }\end{array}$ & CDC \\
\hline EPI706423 & HA & Paraguay & A/Paragua y/6987/2015 & $\begin{array}{c}\text { Laboratorio Central de Salud } \\
\text { Pública }\end{array}$ & CDC \\
\hline EPI706415 & HA & Paraguay & A/Paragua y/6242/2015 & $\begin{array}{c}\text { Laboratorio Central de Salud } \\
\text { Pública }\end{array}$ & CDC \\
\hline E PI643471 & HA & Paraguay & A/Paraguay/1871/2015 & $\begin{array}{c}\text { Laboratorio Central de Salud } \\
\text { Pública }\end{array}$ & CDC \\
\hline E PI643439 & HA & P araguay & A/Paraguay/5333/2015 & $\begin{array}{c}\text { Laboratorio Central de Salud } \\
\text { Pública }\end{array}$ & CDC \\
\hline E PI543034 & $\mathrm{HA}$ & Paraguay & A/Paraguay/64/2014 & $\begin{array}{l}\text { Laboratorio Central de Salud } \\
\text { Pública }\end{array}$ & CDC \\
\hline EPI543028 & HA & Paraguay & A/Paraguay/05/2014 & $\begin{array}{l}\text { Laboratorio Central de Salud } \\
\text { Pública }\end{array}$ & CDC \\
\hline EPI485771 & HA & $P$ araguay & A/Paraguay/91/2013 & $\begin{array}{l}\text { Laboratorio Central de Salud } \\
\text { Pública }\end{array}$ & CDC \\
\hline E PI484969 & HA & P araguay & A/Paraguay/17/2013 & $\begin{array}{l}\text { Laboratorio Central de Salud } \\
\text { Pública }\end{array}$ & CDC \\
\hline EPI394758 & HA & Paraguay & A/Paraguay/20199/2012 & $\begin{array}{l}\text { Laboratorio Central de Salud } \\
\text { Pública }\end{array}$ & CDC \\
\hline EPI387972 & HA & Paraguay & A/Paraguay/114/2012 & $\begin{array}{c}\text { Laboratorio Central de Salud } \\
\text { Pública }\end{array}$ & CDC \\
\hline EPI349352 & HA & Paraguay & A/Paraguay/191/2011 & $\begin{array}{l}\text { Laboratorio Central de Salud } \\
\text { Pública }\end{array}$ & $\mathrm{CDC}$ \\
\hline E PI349349 & HA & Paraguay & A/Paraguay/105/2011 & $\begin{array}{c}\text { Laboratorio Central de Salud } \\
\text { Pública }\end{array}$ & CDC \\
\hline EPI301701 & HA & Paraguay & A/Paragu ay/901/2010 & $\begin{array}{l}\text { Laboratorio Central de Salud } \\
\text { Pública }\end{array}$ & CDC \\
\hline EPI301698 & HA & Paraguay & A/Paraguay/813/2010 & $\begin{array}{c}\text { Laboratorio Central de Salud } \\
\text { Pública }\end{array}$ & CDC \\
\hline EPI183250 & HA & Paraguay & A/Paraguay/09/2009 & $\begin{array}{l}\text { Laboratorio Central de Salud } \\
\text { Pública }\end{array}$ & CDC \\
\hline $\mathrm{EPI} 221023$ & HA & $P$ araguay & A/Paraguay/08/2009 & $\begin{array}{c}\text { Laboratorio Central de Salud } \\
\text { Pública }\end{array}$ & CDC \\
\hline E PI184390 & HA & Paraguay & A/Paraguay/28/2009 & $\begin{array}{l}\text { Laboratorio Central de Salud } \\
\text { Pública }\end{array}$ & CDC \\
\hline
\end{tabular}

\section{Referencias bibliográficas}

1. Suárez $P$, Valcarcel J, Ortín J. Heterogeneity of the mutation rates of influenza A viruses: isolation of mutator mutants. J Virol. 1992;66:2491-4.

2. Itoh $\mathrm{Y}$, Shinya K, Kiso M, Watanabe T, Sakoda $\mathrm{Y}$, Hatta M, et al. In vitro and in vivo characterization of new swine-origin H1N1 influenza viruses. Nature. 2009;460:1021-5.

3. Smith GJ, Vijaykrishna D, Bahl J, Lycett SJ, Worobey M, Pybus OG, et al. Origins and evolutionary genomics of the 2009 swine-origin H1N1 influenza A epidemic. Nature. 2009;459:1122-5.

4. Klimov AI, Garten R, Russell C, Barr IG, Besselaar TG, Daniels R, Engelhardt OG, Grohmann G, Itamura S, Kelso A, McCauley J, Odagiri T, Smith D, Tashiro M, Xu X, Webby R, Wang D, Ye Z, Yuelong S, Zhang W, Cox N. WHO recommendations for the viruses to be used in the 2012 Southern Hemisphere Influenza Vaccine: epidemiology, antigenic and genetic characteristics of influenza $A(H 1 N 1) p d m 09, A(H 3 N 2)$ and $B$ influenza viruses collected from February to September 2011. Vaccine. 2012 Oct 5;30(45):6461-71.

5. Garten RJ, Davis CT, Russell CA, Shu B, Lindstrom S, Balish A, et al. Antigenic and genetic characteristics of swine-origin $2009 \mathrm{~A}(\mathrm{H} 1 \mathrm{~N} 1)$ influenza viruses circulating in humans. Science. 2009;325:197-201. 
6. Girard MP, Tam JS, Assossou OM, Kieny MP. The 2009 A (H1N1) influenza virus pandemic: a review. Vaccine. 2010;28:4895-902.

7. Everitt AR, Clare S, Pertel T, John SP, Wash RS, Smith SE, et al. IFITM3 restricts the morbidity and mortality associated with influenza. Nature. 2012;484:519-23.

8. Hungnes $O$. The role of genetic analysis in influenza virus surveillance and strain characterisation. Vaccine. 2002;20 Suppl 5:B45-9.

9. Tamura K1, Stecher G, Peterson D, Filipski A, Kumar S. MEGA6: Molecular Evolutionary Genetics Analysis version 6.0. Mol Biol Evol. 2013 Dec;30(12):2725-9.

10. Skehel JJ, Wiley DC. Receptor binding and membrane fusion in virus entry: the influenza hemagglutinin. Annu Rev Biochem. 2000;69:531-69.

11. Caton AJ, Brownlee GG, Yewdell JW, Gerhard W. The antigenic structure of the influenza virus A/PR/8/34 hemagglutinin (H1 subtype). Cell. 1982;31(2 Pt 1): 417-27.

12. Winter G, Fields S, Brownlee GG. Nucleotide sequence of the haemagglutinin gene of a human influenza virus $\mathrm{H} 1$ subtype. Nature. 1981;292:72-5.

13. Xu R, Ekiert DC, Krause JC, Hai R, Crowe JE Jr, Wilson IA. Structural basis of preexisting immunity to the $2009 \mathrm{H} 1 \mathrm{~N} 1$ pandemic influenza virus. Science. 2010;328:357-60.

14. Antón A, Pozo F, Niubó J, Casas I, Pumarola T. Influenza A(H1N1)pdm09 virus: viral characteristics and genetic evolution. Enferm Infecc Microbiol Clin. 2012 Oct;30 Suppl 4:10-7.

15. Yang $\mathrm{H}$, Carney $\mathrm{P}$, Stevens J. Structure and receptor binding properties of a pandemic

H1N1 virus hemagglutinin. PLoS Curr. 2010;2:RRN1152.

16. Kilander A, Rykkvin R, Dudman SG, Hungnes O. Observed association between the HA1 mutation D222G in the 2009 pandemic influenza $A(H 1 N 1)$ virus and severe clinical outcome, Norway 2009-2010. Euro Surveill. 2010;15:19498.

17. Childs RA, Palma AS, Wharton S, Matrosovich T, Liu Y, Chai W, et al. Receptorbinding specificity of pandemic influenza A (H1N1) 2009 virus determined by carbohydrate microarray. Nat Biotechnol. 2009;27:797-9

18. Belser JA, Jayaraman A, Raman R, Pappas C, Zeng H, Cox NJ, et al. Effect of D222G mutation in the hemagglutinin protein on receptor binding, pathogenesis and transmissibility of the 2009 pandemic H1N1 influenza virus. PLoS One. 2011;6:e25091.

19. Chutinimitkul S, Herfst S, Steel J, Lowen AC, Ye J, Van Riel D, et al. Virulence-associated substitution D222G in the hemagglutinin of 2009 pandemic influenza $A(H 1 N 1)$ virus affects receptor binding. J Virol. 2010;84:11802-13.

20. Recommended composition of influenza virus vaccines for use in the 2016-2017 northern hemisphere influenza season. Wkly Epidemiol Rec. 2016;91:121-32. https://www.ncbi.nlm.nih.gov/pubmed/26971356

21. WHO. Recommended composition of influenza virus vaccines for use in the 2017 southern hemisphere influenza season. http://www.who.int/entity/influenza/vaccines/virus/ recommendations/201609_recommendation.pdf?ua=1 